\title{
VESSEL MOTIONS AND WORK INTERRUPTIONS ABOARD A FAST RESCUE CRAFT
} (DOI No: 10.3940/ijsct.2021.a1.236)

M Garvin and T Harris, National Research Council, Canada

KEY DATES: Submitted: 15/06/2020, Final acceptance: 08/12/2020, Published: 25/03/2021

\section{SUMMARY}

A set of field trials were carried out aboard a Canadian Coast Guard fast rescue Rigid Hull Inflatable Boat. The vessel was outfitted with a data acquisition system to collect vessel and engine performance data and trialled in three wave conditions (approx. Beaufort 2 to 7). This paper focusses on the methodologies and results for calculating and investigating MotionInduced Interruptions (MIIs). MIIs due to lateral and longitudinal overbalancing and sliding were investigated using the counting of motion events which are expected to cause an interruption, supported by a statistical analysis and examination of the distribution of the MII data. We conclude that MII assessments of small, light, high-speed craft such as the one studied should include longitudinal acceleration and pitch angle, typically assumed to be non-influential in MII assessments. Statistical treatments have promise for analysis of field-acquired MII data.

\section{NOMENCLATURE}

$b \quad$ Half-stance width of a person standing on the deck of the vessel [m]

d Halffoot length of a person standing on the deck of the vessel [m]

$g \quad$ Acceleration due to gravity $(9.81 \mathrm{~m} / \mathrm{s} 2)$

$h \quad$ Distance from the deck to the centre of gravity of a person standing on the deck of the vessel [m]

MII Motion-Induced Interruption

$S_{a} \quad$ Longitudinal sliding force, aft, normalized to threshold value

$S_{f} \quad$ Longitudinal sliding force, forward, normalized to threshold value

$S_{\text {Lat }}(t)$ Dominant lateral sliding force at time $t$, normalized to threshold value

$S_{\text {Long }}(t)$ Dominant longitudinal sliding force at time $t$, normalized to threshold value

$S_{P} \quad$ Lateral sliding force, to Port, normalized to threshold value

$S_{S} \quad$ Lateral sliding force, to Starboard, normalized to threshold value

$T_{a} \quad$ Longitudinal tipping moment, aft, normalized to threshold value

$T_{f}$ Longitudinal tipping moment, forward, normalized to threshold value

$T_{\text {Lat }}(t)$ Dominant lateral tipping moment at time $t$, normalized to threshold value

$T_{\text {Long }}(t)$ Dominant longitudinal tipping moment at time $t$, normalized to threshold value

$T_{P} \quad$ Lateral tipping moment, to Port, normalized to threshold value

$T_{S} \quad$ Lateral tipping moment, to Starboard, normalized to threshold value

$\eta_{1,2,3} \quad$ Motion in the $x$ (surge), $y$ (sway), and $z$ (heave) directions $[\mathrm{m}]$

$\eta_{4,5,6} \quad$ Rotation about the $x$ (surge), $y$ (sway), and $z$ (heave) axes [radians]

$\mu \quad$ Coefficient of friction between footwear and the deck of the vessel

\section{INTRODUCTION}

Effectiveness of work at sea is not only determined by the performance of the equipment being used and the intrinsic skills of the operators; the effects of vessel motion and vibration on the crew's ability to perform to their normal capacity must also be considered. The physiological effects of vessel motion and vibration are diverse. A description of the range of effects, from motion sickness and fatigue to more acute health and safety effects is provided in (Matsangas et al., 2009). Operations at sea provide a unique challenge; if conditions deteriorate it is often not possible to seek respite; work must continue through adverse conditions. This is particularly true of emergency response at sea where mission effectiveness is expected in all weather conditions.

The work discussed herein developed from a series of test aboard a Canadian Coast Guard Fast Rescue Craft (FRC). The initial goal of the tests was to assess the performance of a set of new spark-ignited diesel outboard engines for use aboard the FRC fleet.

\section{METHODS}

The evaluation included a set of star pattern tests completed in three different wave conditions at five speeds which form the basis for this paper.

Facilitating the test were a group of experienced Search and Rescue FRC operators. Conversations with these operators revealed a physiological and psychological toll from long-term exposure to Search and Rescue FRC operations which, by definition, occur in all types of weather and involve pushing boats and crews to their performance limit. Absent specific human-factors data, the task of assessing the difficulty of working aboard the FRC from available data was undertaken. To assess the physiological challenges facing crew aboard an FRC, it was decided to assess the degree to which whole-body 
vessel motions impact the crew's ability to perform tasks. The metric used for this assessment is Motion-Induced Interruptions (MIIs).

MII was chosen since vessel operators are position themselves in a unique stance while operating the vessel. The typical posture observed aboard the vessel had the crew effectively standing while holding partial body weight above a horse saddle-like seating arrangement. While this differs from the classical application of the MII calculation which identifies motions that would cause a standing person to slide or lose balance (Baitis et al., 1984; Graham, 1990), it is appropriate in this case since the operators are bearing most of their weight on their feet and a motion severe enough to cause a slide or loss of balance will force the operators to steady themselves with their legs or hands. While this steadying prevents a fall, it has potential to distract the crew from their task or limit their effectiveness as some of their energy and attention is devoted to stabilizing themselves. The incidence of MII, then (as previously defined), provides a basis for comparing the relative level to which the crew is distracted or has their energy and attention diverted from their primary task.

A MII is defined as a combination of vessel motions that would cause a standing person to lose postural control either by sliding on the deck surface or overbalancing either forward/aft or side to side, thus causing the individual to temporarily cease work as they stabilize themselves. An overview of the typical application of motion-induced interruption (MII) calculations is presented in (Gaglione et al., 2016). In the case of the sliding mode, these events occur when forces due to vessel motions exceed the frictional force between the person's feet and the deck. In the case of the tipping mode, these events occur when the moments of forces acting parallel to the deck about the person's foot exceed the moments of forces acting perpendicular to the deck about the person's foot. The thresholds at which sliding occurs are related to the force of gravity and the coefficient of friction between the person's foot and the deck. The thresholds for tipping include the half-width of the person's stance (lateral) and the half-length of the person's foot (longitudinal). For ease of interpretation, MII data presented herein is normalised to the threshold value presented in (Gaglione et al., 2016).

The sliding forces at a hypothetical Point $Q$ to port $(P)$ and to starboard $(S)$, normalized to the threshold value can be calculated using Equation 1 and 2. Sliding is indicated by $S_{P} \vee S_{S} \geq 1$.

$$
\begin{gathered}
S_{P}=\frac{-\ddot{\eta}_{2 Q}-g \eta_{4}-\mu \ddot{\eta}_{3 Q}}{\mu g} \\
S_{S}=\frac{+\ddot{\eta}_{2 Q}+g \eta_{4}-\mu \ddot{\eta}_{3 Q}}{\mu g}
\end{gathered}
$$

The forward $(f)$ and aft $(a)$ longitudinal moments at Point $Q$, normalized to the threshold can be calculated using Equation 3 and 4. Longitudinal tipping is indicated $\operatorname{by}_{f} \vee T_{a} \geq 1$.

$$
\begin{aligned}
T_{f} & =\frac{-\frac{1}{3} h \ddot{\eta}_{5}-\frac{d}{h} \ddot{\eta}_{3 Q}}{\frac{d}{h} g} \\
T_{a} & =\frac{+\frac{1}{3} h \ddot{\eta}_{5}-\frac{d}{h} \ddot{\eta}_{3 Q}}{\frac{d}{h} g}
\end{aligned}
$$

The port $(P)$ and starboard $(S)$ lateral moments at Point $Q$, normalized to the threshold value can be calculated using Equation 5 and 6. Lateral tipping is indicated by $T_{P} \vee T_{S} \geq 1$.

$$
\begin{aligned}
& T_{P}=\frac{+\frac{1}{3} h \ddot{\eta}_{4}-\ddot{\eta}_{2 Q}-g \eta_{4}-\frac{b}{h} \ddot{\eta}_{3 Q}}{\frac{b}{h} g} \\
& T_{S}=\frac{-\frac{1}{3} h \ddot{\eta}_{4}+\ddot{\eta}_{2 Q}+g \eta_{4}-\frac{b}{h} \ddot{\eta}_{3 Q}}{\frac{b}{h} g}
\end{aligned}
$$

Where:

$\eta_{1}, \eta_{2}, \eta_{3},\left(\eta_{4}, \eta_{5}, \eta_{6}\right)$ are the motions (rotations) along (around) the ship longitudinal, transversal and vertical axes, respectively, as shown in Figure 1.

$\mu$ is the coefficient of friction between the deck and the operators' footwear, assumed to be 0.7 (Baitis et al., 1984)

$\frac{d}{h}$ is the ratio between the half foot length and the operators' vertical center of gravity, assumed to be 0.17 (Baitis et al., 1984)

$\frac{b}{h}$ is the ratio between the half stance width and the operators' vertical centre of gravity, assumed to be 0.25 (Baitis et al., 1984)

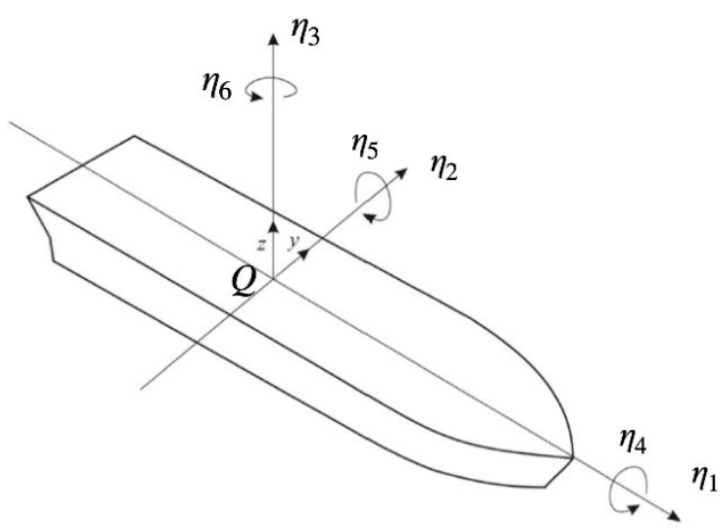

Figure 1: Coordinate system used throughout the paper. 
The rate of MIIs can be used to assess the risk presented by the motion. Based on the discussion in (Graham, 1990), the risk of sliding or tipping is assigned a descriptor of the risk level as shown in Table 2. These thresholds were converted into a rate of MII per hour to facilitate visualization.

Table 1: MII risk level descriptors and thresholds.

\begin{tabular}{|l|l|l|}
\hline $\begin{array}{l}\text { Risk Level } \\
\text { Descriptor }\end{array}$ & $\begin{array}{l}\text { MII per minute } \\
\text { (Graham, 1990) }\end{array}$ & MII per hour \\
\hline "Possible" & $>0.1$ & $>6$ \\
\hline "Probable" & $>0.5$ & $>30$ \\
\hline "Serious" & $>1.5$ & $>90$ \\
\hline "Severe" & $>3.0$ & $>180$ \\
\hline "Extreme" & $>5.0$ & $>300$ \\
\hline
\end{tabular}

It is important to highlight that the MII calculations discussed above are premised on a person in a standing position and facing forward. No prior work was found that addressed similar interruptions to work for people in sitting positions. (Crossland et al., 2007) conducted a series of tests that measured MIIs in participants performing a variety of tasks involving postural changes although the participants were predominantly standing. The crew position aboard the FRC is a hybrid between sitting and standing; the crewmember straddles a saddlestyle seat, facing forward, with their weight shared between their feet and the seat. The seat and abundant handholds around the crew positions mean that crew are much more able to steady themselves and are unlikely to slide or tip as modelled by the MII calculations. The MII calculations do, however, provide a reference by which to judge the relative severity of the motion. While crew in a semi-seated position with abundant handholds may not actually slide or tip in response to the motions, much of their energy and attention may be needed to maintain their posture by, for instance, holding on with one hand or gripping the seat with their legs. This, in turn, diminishes their capacity to undertake other tasks. Furthermore, the nature of Search and Rescue missions is unpredictable and crew may be required to move about the FRC for various reasons during a mission.

\section{TRIALS EQUIPMENT}

The test vessel was a Zodiac Hurricane 753 OB Rigid-Hill Inflatable Boat (RHIB) in Search and Rescue configuration with a pair of Mercury Marine DSI 3.0 spark-ignited diesel outboard motors, rated at $130 \mathrm{~kW}$ each. In this configuration, the helmsman's console is centreline, approximately amidships with navigator consoles to port and starboard of centreline immediately aft of the helmsman (Zodiac Hurricane Technologies, Inc., 2015). All three consoles include saddle-style seats with shock absorbers. Throughout the test, three crewmembers (two SAR operators + one researcher) maintained the same nominal positions. The helmsman's controls include dual throttles and a steering wheel. The vessel has dual (forward and aft) fuel tanks which were filled prior to each test day. The vessel's principal particulars and pertinent details are listed in Table 2.

Table 2: Principal particulars of the test vessel (Zodiac Hurricane Technologies, Inc., 2015).

\begin{tabular}{|l|l|}
\hline Overall Length & $8.15 \mathrm{~m}$ \\
\hline Beam & $2.75 \mathrm{~m}$ \\
\hline Displacement (as tested) & $2,837 \mathrm{~kg}$ \\
\hline $\begin{array}{l}\text { Longitudinal Centre of Gravity } \\
\text { (as tested) }\end{array}$ & $2.32 \mathrm{~m}$ \\
\hline $\begin{array}{l}\text { Vertical Centre of Gravity } \\
\text { (as tested) }\end{array}$ & $0.51 \mathrm{~m}$ \\
\hline Fuel Capacity & $3211(241 \mathrm{~kg}) \mathrm{aft}$, \\
& $1811(136 \mathrm{~kg}) \mathrm{fwd}$. \\
\hline
\end{tabular}

Throughout the test series, the FRC was fitted with a data acquisition system, providing a comprehensive record of the vessel's performance. During the star pattern tests, a wave buoy was deployed in the test area to capture wave condition information. Data collected included vessel speed and course (DGPS), and accelerations / rotational rates inside the operator's console (accelerometer / rate gyroscope combination). All data was sampled at $100 \mathrm{~Hz}$.

For the first two wave conditions tested, a TRYAXIS ${ }^{\mathrm{TM}}$ Directional Wave Buoy (AXYS Technologies Inc., n.d.) was moored near the test site. Since the third wave condition was tested in deep water, mooring was not feasible so a TRYAXIS $^{\mathrm{TM}}$ Mini Wave Buoy (AXYS Technologies Inc., n.d.) was connected to a drogue and allowed to drift downwind through the test area during the test.

\section{PROCEDURES}

Testing was conducted in three wave conditions. Each test consisted of a series of straight legs at different courses relative to the wave direction. The test areas for wave condition 1 and 2 were located in Conception Bay in Eastern Newfoundland, to the West and Southwest, respectively, of Bell Island. The test area for wave condition 3 was located off St. John's in Eastern Newfoundland. The target courses relative to wave direction were: $0,45,90,120,150,180,225,270,300$, and 330 degrees $(0$ degrees indicating head seas, 90 degrees indicating starboard beam seas, etc.). The duration of each leg was adjusted based on the target boat speed, with slower speeds maintained for a longer duration. The star patterns were planned with target boat speeds of (leg duration in brackets): 4 knots (240 seconds), 8 knots ( 240 seconds), 20 knots (180 seconds), 30 knots (120 seconds), and full speed $/ 38$ knots ( 90 seconds). In head seas, this test program yielded at least 50 wave encounters for each speed (Seakeeping Committee, 28TH ITTC, 2017). Each test course was steered once at each speed and wave state.

For wave condition 1, the wind was less than 5 knots with waves entering the mouth of the bay from open ocean as shown in Figure 2. For wave condition 2, the wind was approximately 20-25 knots, in line with the wave 
direction. Waves were primarily wind-driven with a short fetch of about 5 nautical miles, as shown in Figure 2. For wave condition 3 , the wind was approximately 15 knots, 30 to 50 degrees offset counter clockwise from the wave direction. Wave condition 3 was tested near shore but in a stretch of water exposed to the open ocean, as shown in Figure 3. Current in the test areas is negligible.

In the first wave condition, the 30 knot and 38 knot star tests were not completed due to concerns about the vessel becoming airborne and experiencing propeller ventilation in the short, steep waves; thus defining the upper edge of the vessel's speed performance envelope in these conditions. In the third wave condition, testing was only completed at 4 knots and 20 knots; higher speeds exceeded the vessel's speed performance envelope for the wave condition and testing was cut short due to safety concerns. Wave buoy data from the duration of the testing was analysed using the wave buoys' on board software to calculate wave statistics: significant wave height, peak period, and steepness for each wave condition tested (Miles et al., 2003). The test wave conditions are summarized in Table 3.

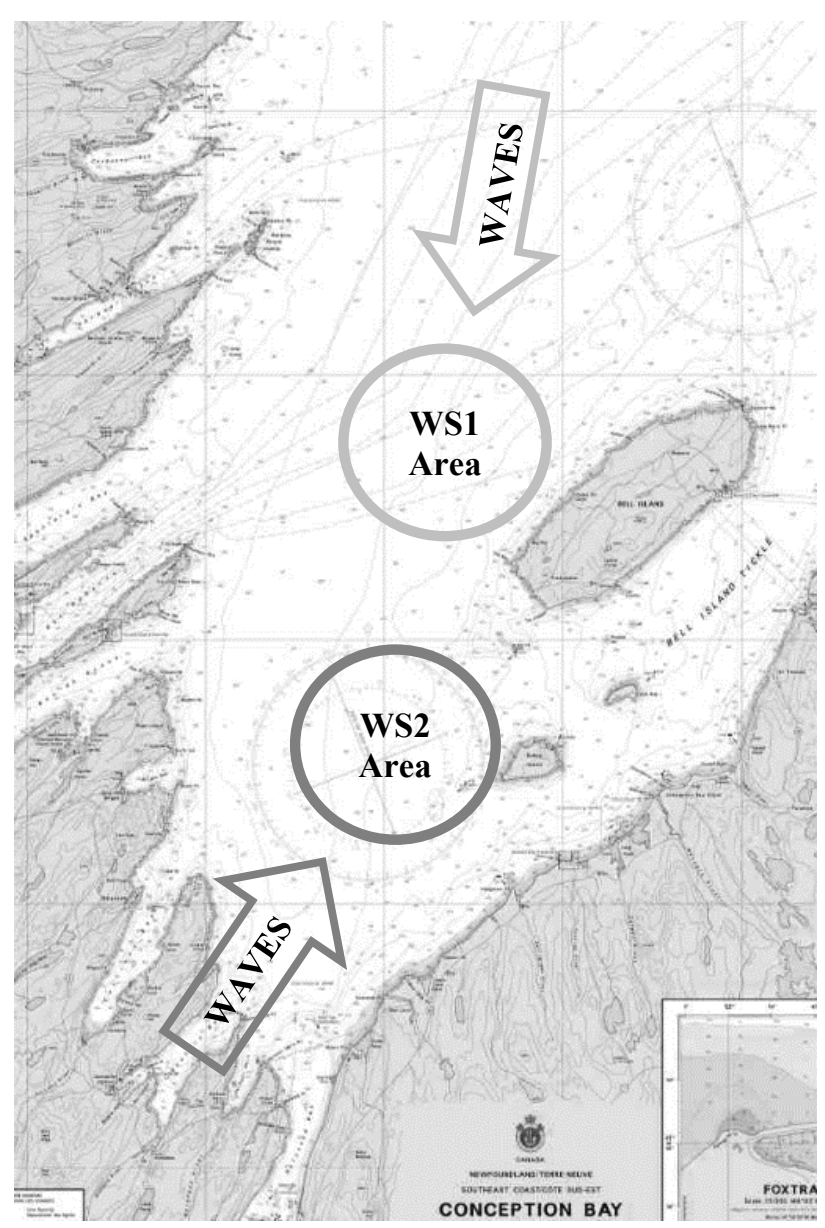

Figure 2: Approximate test areas for wave conditions 1 and 2, with approximate wave direction. Excerpt from (Canadian Hydrographic Service, 2001).

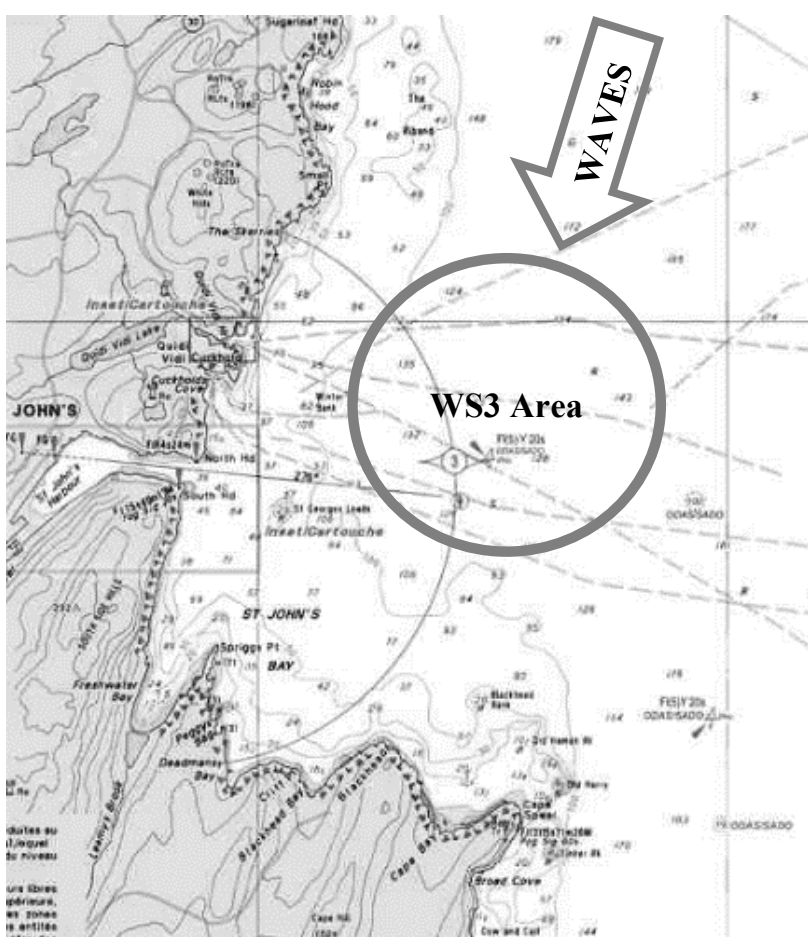

Figure 3: Approximate test area for wave condition 3, with approximate wave direction. Excerpt from (Canadian Hydrographic Service, 1995).

Table 3: Summary of test wave conditions.

\begin{tabular}{|l|l|l|l|}
\hline & $\begin{array}{l}\text { Significant } \\
\text { Height }\end{array}$ & $\begin{array}{l}\text { Peak } \\
\text { Period }\end{array}$ & Steepness \\
\hline wave condition 1 & $0.40 \mathrm{~m}$ & $6.51 \mathrm{~s}$ & 0.077 \\
\hline wave condition 2 & $0.52 \mathrm{~m}$ & $2.71 \mathrm{~s}$ & 0.67 \\
\hline wave condition 3 & $3.6 \mathrm{~m}$ & $11.9 \mathrm{~s}$ & 0.52 \\
\hline
\end{tabular}

Where,

$$
\text { Wave Steepness }=\frac{\text { Wave Height }}{\text { Wave Length }}
$$

The motion data used in analysis is from a location in the FRC helmsman's console ( $0.5 \mathrm{~m}$ forward of amidships, on centreline, and level with the deck surface). This was considered to be a reasonable approximation of the motions of the deck experienced by the operator (within $0.2 \mathrm{~m}$, depending on the position of the helmsman's feet). The motions at the navigators' consoles (approximately $1 \mathrm{~m}$ aft) were not specifically considered.

Since MII primarily occur at frequencies lower than $1 \mathrm{~Hz}$ (Matsangas et al., 2009; Colwell, 1989), a low-pass filter was applied to the data to remove high-frequency vibrations above $1 \mathrm{~Hz}$. Next, the MII values were calculated at each time step using Equations 1 thru 6. At each time $(t)$, the largest of each pair of MII values was added to the dataset for Sliding, Longitudinal Tipping, and Lateral Tipping, effectively building a time series of the sliding forces and tipping moments, normalized to the threshold, as shown in Equation 7 to 9. 


$$
\begin{aligned}
& S_{\text {Lat }}(t)= \begin{cases}S_{S}(t), & S_{P}(t)<S_{S}(t) \\
S_{P}(t), & S_{P}(t) \geq S_{S}(t)\end{cases} \\
& T_{\text {Long }}(t)= \begin{cases}T_{f}(t), & T_{a}(t)<T_{f}(t) \\
T_{a}(t), & T_{a}(t) \geq T_{f}(t)\end{cases} \\
& T_{\text {Lat }}(t)= \begin{cases}T_{S}(t), & T_{P}(t)<T_{S}(t) \\
T_{P}(t), & T_{P}(t) \geq T_{S}(t)\end{cases}
\end{aligned}
$$

Up-crossings through the threshold were used to detect a MII by comparing data at adjacent time steps. MIIs detected in this way were counted for each dataset and divided by the duration of the dataset to yield the MII occurrence rate (MIIs per hour).

Initially, the incidence of MII was calculated on the full dataset, representative of the full range of speeds, headings, and wave conditions that the FRC might experience during an operation. Over the full dataset, there were 14 incidents of lateral sliding per hour, 196 incidents of lateral tipping per hour, and 0 incidents of longitudinal tipping per hour.

In previous literature (Gaglione et al., 2016; Graham, 1990), an assumption is made that longitudinal accelerations are small compared to transverse and vertical accelerations and can therefore be neglected in the estimates of MII. Similarly, pitch angle is not considered in the longitudinal tipping equations. From experience aboard the FRC, longitudinal and pitching motions can be quite violent. For this reason, Equations 1, 2, and 7 were used as a basis to calculate longitudinal (forward, $f$, and aft, a) sliding forces, normalized to the threshold value as shown in Equation 10 and 11 with sliding indicated by $S_{f} \vee S_{a} \geq 1$.

$$
\begin{aligned}
& S_{f}=\frac{-\ddot{\eta}_{1 Q}-g \eta_{5}-\mu \ddot{\eta}_{3 Q}}{\mu g} \\
& S_{a}=\frac{+\ddot{\eta}_{1 Q}+g \eta_{5}-\mu \ddot{\eta}_{3 Q}}{\mu g}
\end{aligned}
$$

The time series for longitudinal sliding forces normalized to the threshold was calculated using Equation 12.

$$
S_{\text {Long }}(t)=\left\{\begin{array}{l}
S_{f}(t), S_{a}(t)<S_{f}(t) \\
S_{a}(t), S_{a}(t) \geq S_{f}(t)
\end{array}\right.
$$

Similarly, Equations 3 and 4 were updated to include longitudinal acceleration $\left(\ddot{\eta}_{1 P}\right)$ and pitch angle $\left(\eta_{5}\right)$, providing the following relationships for longitudinal $(f$ and $a$ ) tipping moments as shown in Equations 13 and 14.

$$
T_{f}=\frac{-\frac{1}{3} h \ddot{\eta}_{5}+\ddot{\eta}_{1 Q}+g \eta_{5}-\frac{b}{h} \ddot{\eta}_{3 Q}}{\frac{b}{h} g}
$$

$$
T_{a}=\frac{+\frac{1}{3} h \ddot{\eta}_{5}-\ddot{\eta}_{1 P Q}-g \eta_{5}-\frac{b}{h} \ddot{\eta}_{3 P Q}}{\frac{b}{h} g}
$$

Calculations using the modified equations show that the incidence of longitudinal tipping is significantly increased to 118 incidents per hour for the full dataset when these factors are considered and longitudinal sliding, while not frequently exceeding the threshold, shows 2 incidents per hour. The revised equation set including the additional longitudinal components is used throughout the remainder of the paper.

In work discussing MII, it is common to present the data as a simple count of the number of times the threshold is exceeded in one hour (Matsangas et al., 2009; Gaglione et al., 2016; Baitis et al., 1984; Graham, 1990). While an efficient way of assessing the risk of motions interrupting work, the single numerical result loses some nuance. For instance, the thresholds are based on a number of assumptions regarding individuals' body proportions, level of training or acclimatization to the environment, and the coefficient of friction between footwear (which varies by type) and the deck (which varies with surface treatment, wetness, contamination, etc.) (Baitis et al., 1984). The use of exceedance of a threshold as the sole criterion misses events that fall just short of the threshold. Consider, for instance, a regular sinusoidal motion that generates a consistent MII value just below the threshold. While a simple count of the number of events exceeding the threshold would show that this motion causes no interruption, variations in body proportions, training and acclimatization, footwear, deck condition, etc. could mean that this motion causes a significant number of interruptions. Rather than consider simply the number of exceedances of the threshold, the distributions of the MII datasets were examined to provide insight into the prevalence of events that are close to the threshold, in addition to the number of occurrences that exceed the threshold.

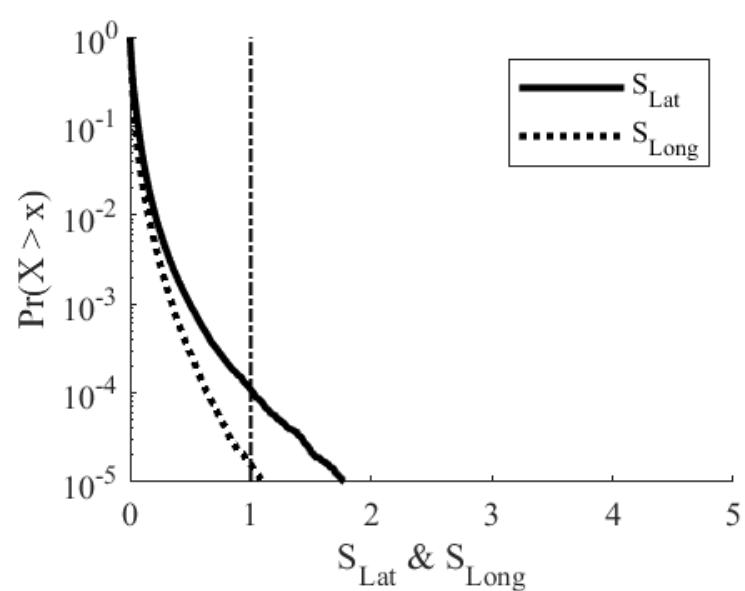

Figure 4: Probability distribution of sliding forces normalized to threshold for the full dataset. Values $>1$ indicate tipping. 
Probability distribution functions of the tipping moments and sliding forces, normalised to the threshold, with the inclusion of longitudinal acceleration and pitch angle, for the full dataset (all wave conditions, headings, and speeds) are shown in Figure 4 and Figure 5. Attempts were made to fit the data to a wide range of parametric distributions but the quality of fit was lower than desired. A nonparametric empirical probability distribution is used to visualize the data.

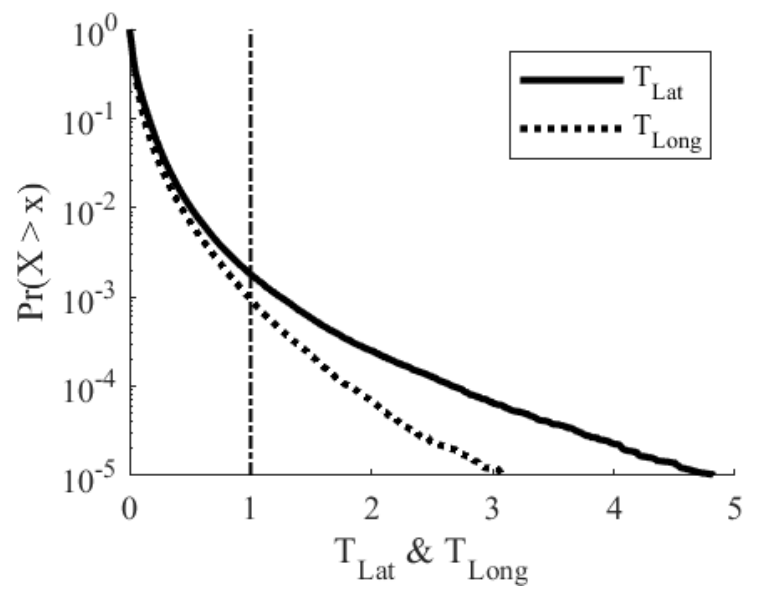

Figure 5: Probability distribution of tipping moments normalized to threshold for the full dataset. Values $>1$ indicate tipping.

\section{RESULTS}

MII occurrence rates are expected to be influenced by wave condition and heading angle relative to wave direction (Gaglione et al., 2016). For high speed craft, we also expect that speed will influence MII occurrence rates. To compare the effect of wave condition and heading, all data were grouped by wave condition $(1,2$, or 3$)$ and target Course Relative to Wave $(0,45,90,120,150,180,225$, 270,300 , and 330 degrees). The standard convention of the author's resident laboratory is 0 degrees head seas, and increasing positively to starboard. Since the full range of speeds was only completed in wave condition 1, data was separated by wave condition and the effect of speed was assessed individually for each wave condition based on the rate of occurrence of MII. These data are presented in Table 4. Plots of data for $T_{\text {Lat }}$ (typically the most severe MII mode) are also provided in Figure 6 to Figure 8 showing the relationship between MII occurrence and wave condition, Course, and Speed. Plots showing the relationship for speed are only provided for wave condition 1 as this covered the full range of speeds. Plots for other MII modes show a similar character. The figures include a line plot of the number of MII occurrences per hour and a scatter plot of the MII value over the threshold. The scatter plots are presented with "jitter" (a random offset either side of the $\mathrm{x}$-axis value sized according to the number of points with similar y-axis value) to provide visual indication of the distribution of the data.

Looking at the full dataset, which is representative of the range of conditions the vessel may experience during a typical mission, lateral tipping MII occurrence is in the "Severe" category with Longitudinal MII occurrence in the "Probable" category. Sliding MII occurrence is much lower ("Minimal" to "Possible").

In general, tipping MIIs are most prevalent, accounting for an order of magnitude more interruptions than sliding MIIs. Lateral MIIs are approximately twice as prevalent as longitudinal MIIs. Longitudinal sliding MIIs (overall the least prevalent mode) are most frequent in wave condition 2 in bow quartering waves where slamming events were most common as described in (Garvin and Harris, 2020) but are rare or non-existent in other conditions.

Looking at the full wave condition datasets, wave condition 1 (the smallest, least-steep waves) produces the lowest occurrence of all modes of MII with fewer extreme events and data distributed at lower values. Wave condition 3 (the largest waves) has the highest occurrence of all MII modes with lateral tipping MIIs reaching the "Extreme" level. This is confirmed by subjective observations aboard the vessel.

The occurrence of MIIs in all modes tends to increase with speed. Sliding MIIs are not prevalent enough in wave condition 1 for this trend to be clear but it is apparent in wave condition 2 and wave condition 3 . The occurrence of tipping MII increase rapidly with speed in all wave conditions. The maximum lateral tipping MII occurrence observed was between 800 and 856 tipping MIIs per hour at the highest speeds in Waves States 1, 2 and 3, which means they lie within $7 \%$ of each other. Maximum longitudinal tipping MII occurrences per hour were similar (524 and 555) for wave condition 2 and 3 but the maximum observed in wave condition 1 was noticeably higher at 692 at 38 knots. In other words, wave condition 3 saw an increase of about $6 \%$ of occurrences from wave condition 2 , whereas wave condition 2 saw a decrease of about $24 \%$ when compared to wave condition 1 .

Occurrence of tipping MIIs is highest in bow quartering conditions. This is consistent with data presented in (Garvin and Harris, 2020) and (Harris, 2018) which indicate that the most extreme motions and difficulty maintaining course/speed were seen in quartering waves. Lateral tipping MIIs reach the "Extreme" level in bow quartering waves across the wave conditions tested. Including data from all three wave conditions, occurrences of MII that range from "Probable" to "Extreme" are seen at all headings in both tipping modes. 
Table 4: Summary of observed MII incidences per hour (rounded to nearest whole-number).

\begin{tabular}{|c|c|c|c|c|c|}
\hline & \multicolumn{2}{|l|}{ Lateral } & \multicolumn{2}{|c|}{ Longitudinal } \\
\hline & & Sliding & Tipping & Sliding & Tipping \\
\hline \multicolumn{2}{|c|}{ Full Dataset } & 14 & 196 & 2 & 118 \\
\hline \multirow{3}{*}{ 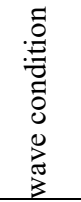 } & 1 & 4 & 122 & 0 & 69 \\
\hline & 2 & 18 & 204 & 5 & 125 \\
\hline & 3 & 43 & 433 & 5 & 275 \\
\hline \multirow{5}{*}{ 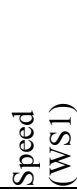 } & 4 & 0 & 0 & 0 & 2 \\
\hline & 8 & 0 & 0 & 0 & 0 \\
\hline & 20 & 0 & 38 & 0 & 6 \\
\hline & 30 & 23 & 337 & 0 & 105 \\
\hline & 38 & 5 & 841 & 0 & 692 \\
\hline \multirow{3}{*}{ 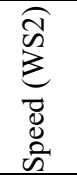 } & 4 & 0 & 2 & 0 & 1 \\
\hline & 8 & 12 & 98 & 9 & 44 \\
\hline & 20 & 53 & 800 & 11 & 524 \\
\hline \multirow{2}{*}{$\begin{array}{l}\vec{D} \tilde{n} \\
\tilde{D} \\
\tilde{n}\end{array}$} & 4 & 0 & 0 & 0 & 0 \\
\hline & 20 & 88 & 856 & 3 & 555 \\
\hline \multirow{10}{*}{$\begin{array}{l}0 \\
\stackrel{6}{3} \\
0 \\
0\end{array}$} & 0 & 5 & 157 & 1 & 136 \\
\hline & 45 & 16 & 302 & 9 & 150 \\
\hline & 90 & 33 & 321 & 12 & 169 \\
\hline & 120 & 12 & 189 & 0 & 108 \\
\hline & 150 & 9 & 120 & 0 & 66 \\
\hline & 180 & 6 & 90 & 0 & 70 \\
\hline & 225 & 4 & 80 & 0 & 52 \\
\hline & 270 & 18 & 169 & 2 & 91 \\
\hline & 300 & 30 & 322 & 0 & 176 \\
\hline & 330 & 41 & 374 & 0 & 251 \\
\hline
\end{tabular}

Shading indicates risk level (Graham, 1990):
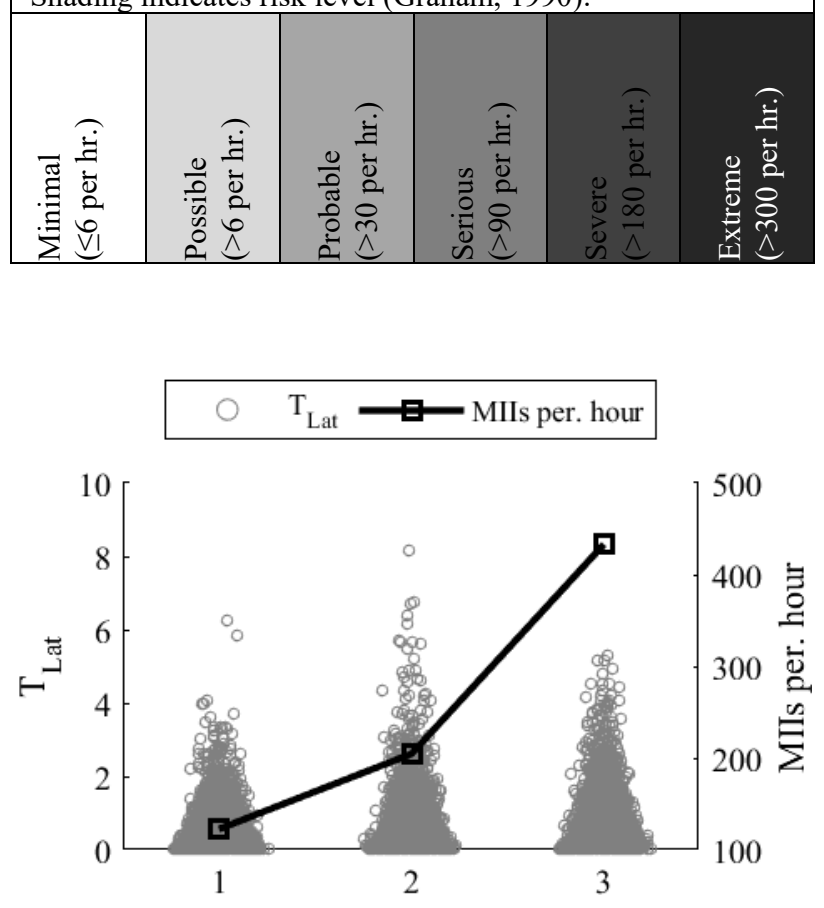

Wave State

Figure 6: $T_{\text {Lat }}$ (values $>1$ indicate tipping) and Lateral Tip MIIs per hour by wave condition, full dataset.

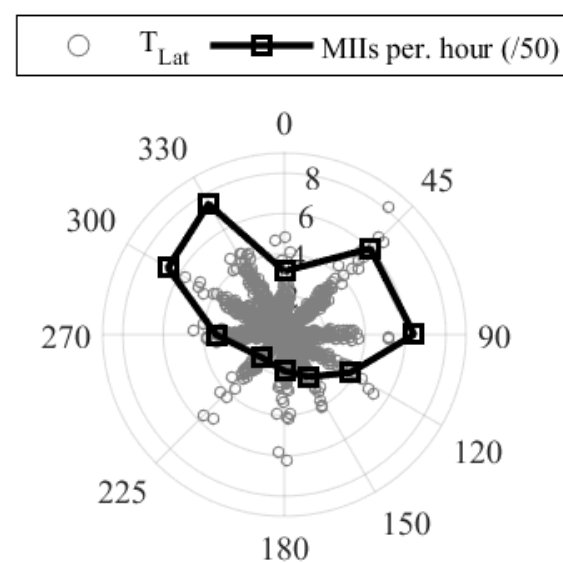

Figure 7: $T_{\text {Lat }}$ (values $>1$ indicate tipping) and Lateral Tip MIIs per hour by Heading Relative to Wave, full dataset.

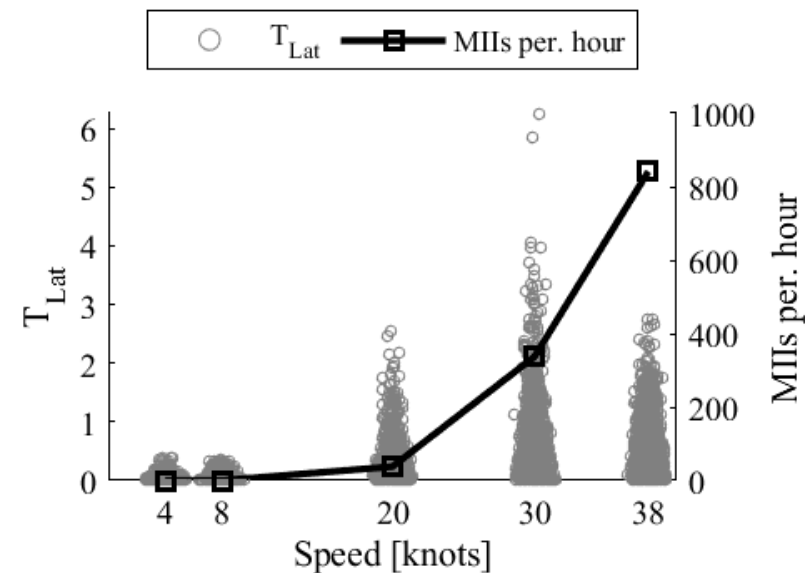

Figure 8: $T_{\text {Lat }}$ (values $>1$ indicate tipping) and Lateral Tip MIIs per hour by Speed, wave condition 1 .

\section{LIMITATIONS}

During the star pattern manoeuvres, it would have been preferable to use a longer leg duration to provide a larger number of wave encounters but the leg length could not be increased given the test speeds and the test area size limitations (approximately 1 nautical mile maximum leg length). Furthermore, there was noticeable crew fatigue with a full star test duration of approximately 2.5 hours during which time the crew experienced significant motions and water spray.

The MII metric used also assumes no interaction from a human to steady oneself due to an interruption. Since the standing posture of those aboard a RHIB is atypical, no source was found that would be more applicable than MII. The authors acknowledge there are other types of work impediment metrics that may apply. Within the scope of this paper, only postural interruptions were explored for relative comparison purposes.

There also may be a discrepancy between what the sensor reads and the motions experienced by the crew due to the 
saddle type shock absorbing seats which cushion vertical accelerations and assist crew steadying.

\section{DISCUSSION}

Differences between the relative appearance of the MII data distribution and the occurrences of MIIs (e.g. 30 knots, and 38 knots in Figure 7) show the importance of looking at the distribution of the MII data rather than just the number of occurrences per hour. In this case, the most extreme events were observed at 30 knots while over twice as many occurrences per hour were observed at 38 knots; a similar phenomenon is seen in Figure 5 for lateral tipping MIIs in wave condition 2 and 3 . In these cases, the dataset showing higher occurrence of MII have a distribution that is 'denser' close to the threshold but fewer extreme events. In essence, there is a larger number of events that exceed the threshold at 38 knots despite events that are more extreme being observed at 30 knots. This phenomenon is explained in Figure 9 which compares the probability distributions at 30 knots and 38 knots in wave condition 1. Extreme events contribute to the rate of MII occurrence (particularly if the rate of MII occurrence is low) but don't significantly change the distribution of the data. This is confounded by differences in the number of observations in the datasets. Wave condition $2(n=720,002)$ contains over twice as many observations as wave condition 3 ( $n=$ 296,680 ). For reference, wave condition 1 contains $n=$ $1,038,005$ data points but their distribution is noticeably lower for all MII modes, resulting in a lower MII occurrence rate. Similarly, the dataset for 30 knots in wave condition $2(n=168,561)$ contains over twice as many observations as 38 knots in wave condition $2(n=$ 74,901). Larger datasets increase the chances that an extreme event will be captured (driving up the MII occurrence rate) but reduce its effect on the distribution.

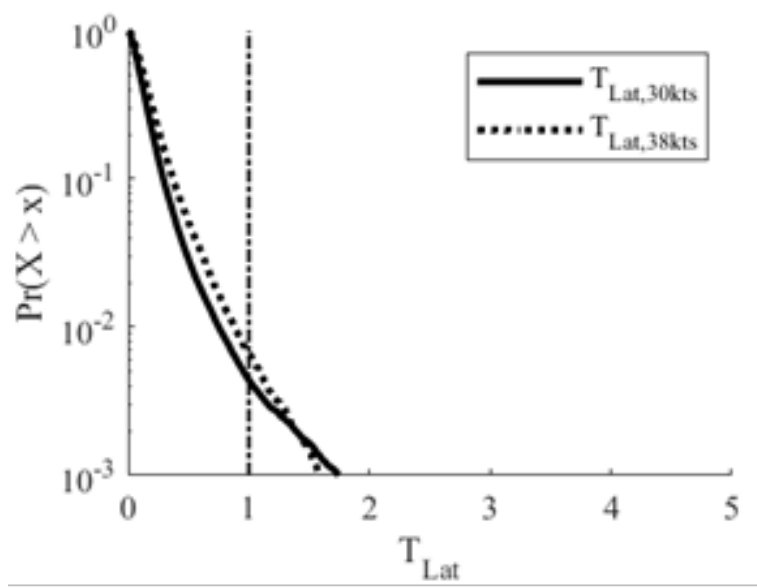

Figure 9. Probability distribution of tipping moments normalized to threshold for 30 knots and 38 knots in wave condition 1 . Values $>1$ indicate tipping.

Examining rare events in measurements of stochastic systems has a potential to lead to erroneous interpretation of data as the rare events may not be reliably captured in limited datasets. Statistical tools and analysis of the distribution of data help to minimize this. Work to assess rare extreme events is a current research topic, specifically examining ship motions in waves using a combination of statistical tools and system dynamics modelling, which may be applicable if more detailed analysis of phenomena such as MII (Mohamad and Sapsis, 2015).

In wave condition 2 and wave condition 3, where the testing was cut short due to safety concerns, it is interesting to note that the MII in lateral and longitudinal tipping, show similar rates of occurrence which are also similar to the highest speed in wave condition 1 . This similarity hints at an upper limit of MII that the boat and crew can tolerate, even with the suspended saddle seat and grab bar design.

The fact that the vessel and crew are able to reliably function in these conditions (particularly the more extreme conditions) is testament to the skill and hardiness of the crew and the design of the vessel's seats and handholds.

\section{CONCLUSIONS}

Assessments of MII on vessels of this type (small, fast, lightweight craft) should include longitudinal acceleration and pitch angle as well as the typically-considered pitch acceleration since the assumption that longitudinal accelerations and pitch angles are small does not hold true. Longitudinal accelerations and pitching motions contribute significantly to MII on this type of vessel, with longitudinal tipping typically the second most prevalent mode of MII. The assumption that longitudinal components are small in comparison to lateral components should be assessed for specific vessels, particularly those with characteristics similar to our test vessel, when engaging in a MII assessment. While field trials provide a definitive assessment of the dominant MII mechanism, previous work (Gaglione et al., 2016) has shown that numerical assessments of MII can be a useful tool that could be applied at the design stage.

These results confirm the crew's subjective opinion of when to slow down and which headings to avoid. This study, however, can be used as an objective guide for operational/response planning. Risk can be minimized by avoiding headings and speeds that result in MII occurrences beyond the level that is considered acceptable. Furthermore, knowledge of the effect of speed and heading on the severity of the resultant vessel motions could be used to optimize speed and heading depending on the specific mission parameters. For example, these results provide evidence of how to modify speed and heading relative to wave to minimize motion severity when transporting an injured person.

MII occurrence rate is based on relatively rare extreme events, assessment from limited stochastic data such as that acquired during field testing is prone to 
misinterpretation especially if the number of occurrences is the only metric examined. Examination of the distribution of the data can provide more insight. This work could be taken further by developing well-fitted distribution models using field data which would allow statistical prediction of MII.

In addition to the relationships previously identified that MII occurrence is affected by wave condition and heading angle relative to wave direction (Gaglione et al., 2016), we can conclude that, at least for this type of lightweight, high-speed planning craft, MII occurrence is also influenced significantly by speed.

\section{ACKNOWLEDGEMENTS}

The authors would like to acknowledge the contribution of the Canadian Coast Guard FRC crew: Neil Peet, Brian Pye, Glen Saunders, and Steve Sheppard. Their enthusiastic professionalism and expertise were crucial to this work. We would also like to acknowledge National Research Council Technical Officer Derek Butler who oversaw the preparation of the test vessel and wave buoys.

\section{REFERENCES}

1. AXYS TECHNOLOGIES INC., n.d. TRIAXYS Directional Wave Buoy. [Online] Available at: https://axystechnologies.com/products/triaxysdirectional-wave-buoy/ [Accessed 1 February 2020].

2. AXYS TECHNOLOGIES INC., n.d. TRIAXYS Mini Wave Buoy. [Online] Available at: https://axystechnologies.com/products/triaxysmini-directional-wave-buoy/ [Accessed 1 February 2020].

3. BAITIS, A. E., APPLEBEE, T. R. et al, 1984. Human Factors Considerations Applied to Operations of the FFG-8 and LAMPS Mk III. Naval Engineers Journal, 97(4), pp. 191-199.

4. CANADIAN HYDROGRAPHIC SERVICE, 1995. Motion Bay to Cape St. Francis Nautical Chart. s.l.: Canadian Hydrographic Service.

5. CANADIAN HYDROGRAPHIC SERVICE, 2001. Conception Bay Nautical Chart. s.l.: Canadian Hydrographic Service.

6. COLWELL, J. L., 1989. Human Factors in the Naval Environment: a Review of Motion Sickness and Biodynamical Problems, Dartmouth: Defence Research Establishment Atlantic.

7. CROSSLAND, P., EVANS, et al., 2007. Motioninduced interruptions aboard ship: Model development and application to ship design. Occupational Ergonomics, Volume 7, pp. 183199.

8. GAGLIONE, S., PISCOPO, V. et al, 2016. The overall motion induced interruptions as operability criterion for fishing vessels. Journal of Marine Science and Technology, Volume 21, pp. 517-532.

9. GARVIN, M. and HARRIS, T., 2020. Operational Assessment of a Fast Rescue Craft. London, Royal Institution of Naval Architects.

10. GRAHAM, R., 1990. Motion-Induced Interruptions as Ship Operability Criteria. Naval Engineers Journal, 102(2), pp. 65-71.

11. HARRIS, T., 2018. Marine Performance Evaluation of a Fast Rescue Craft. St. John's: Master of Engineering Thesis, Memorial University.

12. MATSANGAS, P., MCCAULEY, M. et al, 2009. Human Performance Standards for Ship Motion: a review and a preliminary gap analysis. Stockholm, Maritime Systems and Technology.

13. MILES, M. D., MANSARD, E., et al., 2003. Triaxys Directional Wave Buoy. s.1.: Canadian Coastal Conference.

14. MOHAMAD, M. A. and SAPSIS, T. P., 2015. Probabilistic Description of Extreme Events in Intermittently Unstable Dynamical Systems Excited by Correlated Stochastic Processes. Journal of Uncertainty Quantification, Volume 3, pp. 709-736.

15. SEAKEEPING COMMITTEE, 28TH ITTC, 2017. Recommended Procedures and Guidelines: Seakeeping Experiments. ITTC Quality System Manual, Volume 7.5.

16. ZODIAC HURRICANE TECHNOLOGIES, INC., 2015. CCG SOLAS- H753 Stability Report, Delta, BC: s.n.

17. ZODIAC HURRICANE TECHNOLOGIES, INC., 2015. H753 OB SAR, General Arrangement, Delta, BC: s.n. 